A LETTERS JOURNAL EXPLORING THE FRONTIERS OF PHYSICS

\title{
OFFPRINT
}

\section{Lehmann effect in compensated cholesteric liquid crystals}

\author{
A. Dequidt and P. Oswald \\ EPL, 80 (2007) 26001
}

Please visit the new website www.epljournal.org 


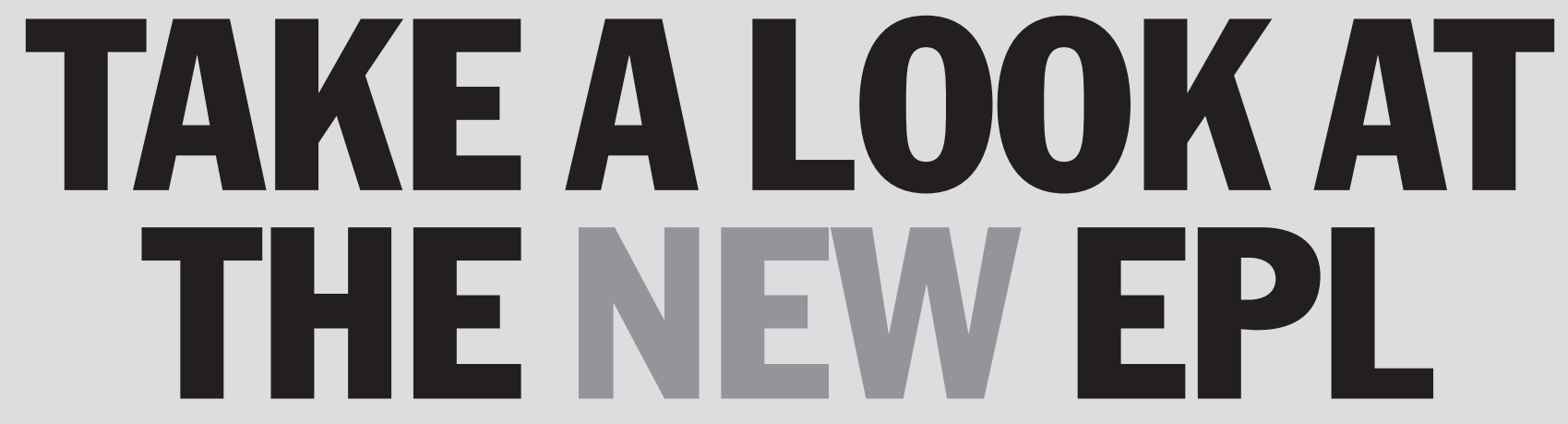

\section{Europhysics Letters (EPL) has a new online home at www.epljournal.org}

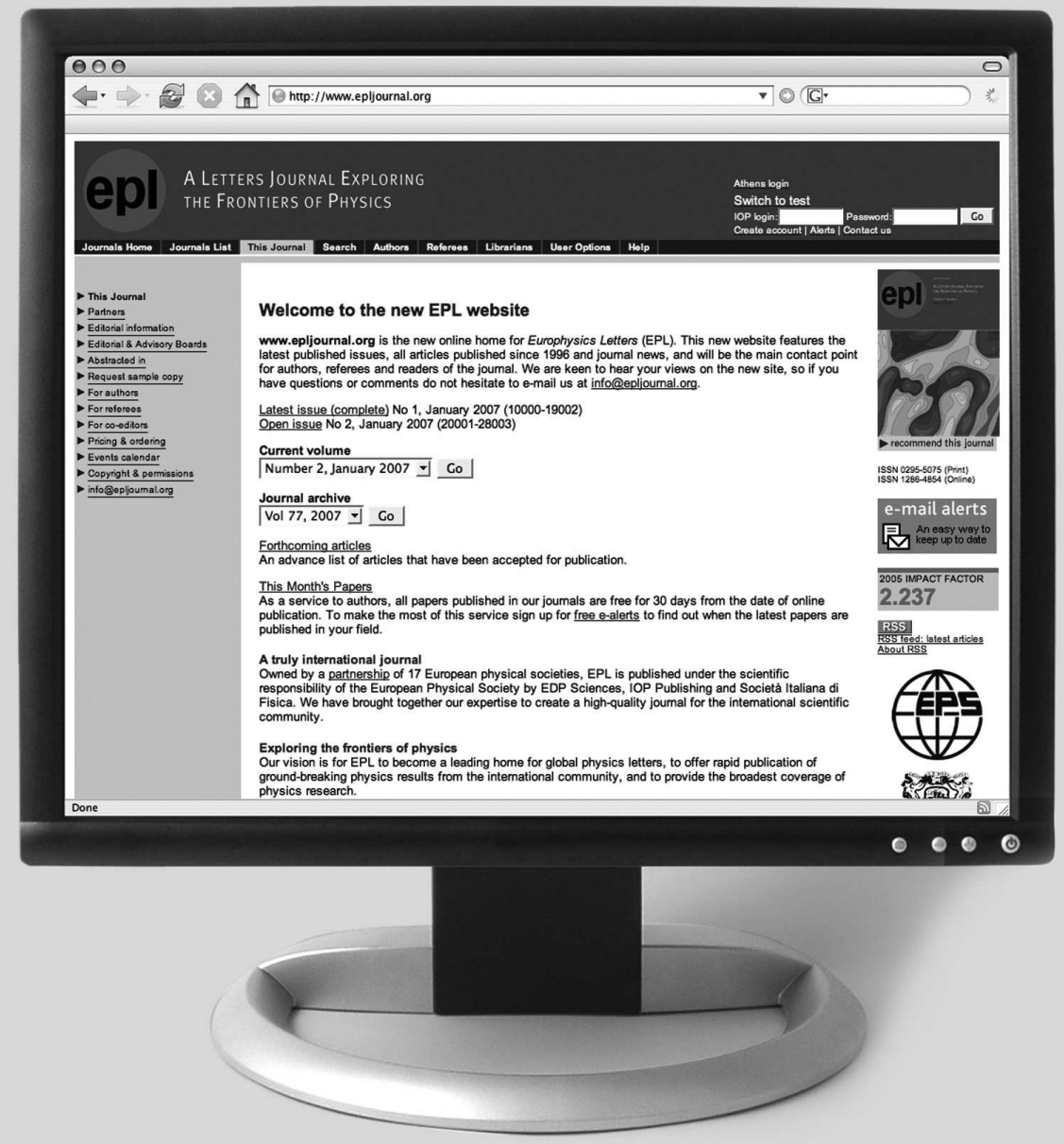

Take a look for the latest journal news and information on:

- reading the latest articles, free!

- receiving free e-mail alerts

- submitting your work to EPL 


\title{
Lehmann effect in compensated cholesteric liquid crystals
}

\author{
A. Dequidt and P. Oswald
}

Laboratoire de Physique, École Normale Supérieure de Lyon, UMR 5672 of the CNRS - 46 Allée d'Italie, 69364 Lyon Cedex 07, France

received 14 July 2007; accepted in final form 1 September 2007

published online 21 September 2007

PACS 61.30.-v - Liquid crystals

PACS 05.70.Ln - Nonequilibrium and irreversible thermodynamics

PACS 65.40.De - Thermal expansion; thermomechanical effects

\begin{abstract}
In 1900, Otto Lehmann observed that the texture of a cholesteric droplet heated from below can rotate continuously (Ann. Phys. (Leipzig), 2 (1900) 649). This observation (which has never been reproduced, to our knowledge) was explained in 1968 by Leslie (Proc. R. Soc. London, Ser. A, 307 (1968) 359) from symmetry arguments accounting for the chirality of the material. In 1982, Éber and Jánossy showed experimentally that a similar thermomechanical effect also exists in a compensated cholesteric (in which the helix is completely unwound). This result was immediately questioned by Pleiner and Brand who claimed that only the symmetry of the phase (and not that of the molecule) determines the structure of the macroscopic constitutive equations (Mol. Cryst. Liq. Cryst. Lett., 5 (1987) 61). According to them, the Lehmann effect should necessarily vanish at the compensation temperature. In order to understand the correct interpretation, we conducted very carefully the experiment in two complementary geometries. Our results agree with those of Éber and Jánossy, confirming the predominance of microscopic symmetries over macroscopic ones.
\end{abstract}

Copyright (C) EPLA, 2007

Introduction. - In 1900, Otto Lehmann observed that particular droplets of a cholesteric liquid crystal spread out between two glass plates could be put into motion when heated from below [1]. This work was completed in a book published in 1921 [2], in which Lehmann clearly showed that it was not the droplet itself, but its optical texture, that was rotating under the influence of the temperature gradient. Surprisingly, the Lehmann experiment has never been reproduced, to our knowledge. The Lehmann rotation was explained qualitatively 68 years later by Leslie [3], who showed that the chirality allows the existence of an internal torque driven by a temperature gradient. More precisely, the Lehmann torque acting on the director $\vec{n}$ reads $[4,5]$

$$
\vec{\Gamma}_{\text {Lehmann }}=-\nu \vec{G}_{\perp}
$$

where $\vec{G}_{\perp}=(\vec{n} \times \vec{G}) \times \vec{n}$ is the component of the temperature gradient $\vec{G}=\vec{\nabla} T$ perpendicular to $\vec{n}$. This equation predicts that the helix of a cholesteric liquid crystal must rotate at a constant angular velocity $\Omega$ when submitted to a temperature gradient parallel to its axis:

$$
\Omega=-\nu G / \gamma_{1}
$$

where $\gamma_{1}$ is the rotational viscosity. Nevertheless, one condition must be satisfied to observe a continuous rotation, which is that the director can freely rotate at the two surfaces limiting the sample. This condition is very difficult to achieve experimentally, which certainly explains why the Lehmann effect is so difficult to reproduce. To overcome this difficulty and measure the Lehmann coefficient $\nu$, Éber and Jánossy performed an ingenious experiment with a cholesteric mixture possessing a compensation temperature, i.e. a temperature at which the equilibrium twist $q=2 \pi / p$ (with $p$ the cholesteric pitch) vanishes and changes sign. Their experiment consisted of measuring the birefringence of a homeotropic sample placed in a temperature gradient perpendicular to the director $\vec{n}[6,7]$. Éber and Jánossy found that, in spite of the fact that the phase has a nematic structure at the compensation point, the director still experiences a Lehmann torque. In other words, they found that $\nu \neq 0$ at the compensation temperature at which $q=0$, concluding that the torque is from microscopic origin and due to the chirality of the molecules. This experiment was immediately criticized by Pleiner and Brand who claimed that the Lehmann coefficient must vanish at the compensation point $[8,9]$. Their argument was the following: "since 
it is the symmetry of the phase which determines the structure of the macroscopic equations, it is clear that the thermomechanical coupling constant has to vanish at the compensation point, since there the symmetry is exactly that of the nematic phase" [9]. They thus concluded that the result of Éber and Jánossy $(\nu \neq 0)$ was wrong and due to some experimental artifact. This affirmation led to a polemic between theorists and experimentalists, the latter claiming that their results were reliable and not forbidden theoretically because of the chirality of the molecules [10]. In view of this controversial situation, we redid the experiment in order to know which of the two types of symmetries, macroscopic or microscopic, was pertinent in the problem. In this letter, we shall only give the results of our measurements, reporting the details of our experiments in a forthcoming longer article.

The plan of the article is the following. In the second section, we recall the principle of the Éber and Jánossy experiment in homeotropic anchoring and we show that it can be extended to the planar geometry. We then calculate for each geometry the director distortions in the temperature gradient and the expression for the phase shift between the ordinary and extraordinary components of a laser beam crossing the sample at the compensation temperature. We show that this shift depends on the optical indices, on the elastic constants and on an effective Lehmann coefficient of expression $\nu_{\text {eff }}=\nu+K_{2} \mathrm{~d} q / \mathrm{d} T$, where $K_{2}$ is the twist Frank constant. In the third section, we describe the experiment and we deduce from the measurement of the optical phase shifts two different combinations of the effective Lehmann coefficient with the optical indices and the two other Frank constants $K_{1}$ and $K_{3}$. In the fourth section, we briefly explain how we measured $\mathrm{d} q / \mathrm{d} T$ as well as the elastic constants $K_{i}$ $(i=1,2,3)$ and the optical indices. Finally, we give in the last section the value of the Lehmann coefficient $\nu$ and we draw conclusions about the role of the molecular chirality.

The Éber and Jánossy experiment: theory. In the original experiment of Éber and Jánossy, the cholesteric liquid crystal is introduced between two parallel glass plates treated for strong homeotropic anchoring. The sample is then placed inside a temperature gradient parallel to the glass plates. In this geometry, the cholesteric helix unwinds when its pitch is typically larger than the sample thickness. As a consequence a band of homeotropic nematic phase, centered on the compensation temperature, forms in the sample. This region is bordered by cholesteric fingers which are well visible under the microscope (for a review about the helix unwinding, see $[5,11]$ ). In practice, the nematic phase is a little distorted because of the presence of the temperature gradient. The director field distortions are obtained by solving the torque equilibrium equations. To first order in temperature gradient $G$, they read

$$
2 K_{2} q \frac{\partial n_{y}(x, z)}{\partial z}+K_{3} \frac{\partial^{2} n_{x}(x, z)}{\partial z^{2}}=0
$$

$$
G \nu_{e f f}+K_{3} \frac{\partial^{2} n_{y}(x, z)}{\partial z^{2}}-2 q K_{2} \frac{\partial n_{x}(x, z)}{\partial z}=0
$$

with $\nu_{e f f}=\nu+\frac{\mathrm{d} K_{2} q}{\mathrm{~d} T}$. The $x$-axis is chosen parallel to the temperature gradient and the $z$-axis perpendicular to the glass plates. The system is invariant along the $y$-direction. Second derivatives with respect to $x$ have been neglected, which can be justified a posteriori (the sample thickness $d$ is always much smaller than the width of the nematic band). Solving the previous equations give:

$$
\begin{gathered}
n_{x}=\frac{G \nu_{e f f}}{2 K_{2} q} d\left(\frac{z}{d}-\frac{1}{2}+\frac{1}{2} \frac{\sin \left(q(d-2 z) \frac{K_{2}}{K_{3}}\right)}{\sin \left(q d \frac{K_{2}}{K_{3}}\right)}\right), \\
n_{y}=\frac{G \nu_{e f f}}{2 K_{2} q} d \frac{\sin \left(q z \frac{K_{2}}{K_{3}}\right) \sin \left(q(d-z) \frac{K_{2}}{K_{3}}\right)}{\sin \left(q d \frac{K_{2}}{K_{3}}\right)} .
\end{gathered}
$$

These equations generalize the solution given by Éber and Jánossy [6] since they are still valid out of the compensation point $T_{c}$. In particular, they give back the spinodal limit for the nematic phase as $n_{x}$ and $n_{y}$ diverge when $q d=\pi\left(K_{3} / K_{2}\right)$ or $d / p=K_{3} /\left(2 K_{2}\right)$.

The solution can be linearized in $q$ in the vicinity of the compensation temperature (at which $q=0$ ), which gives

$n_{x}=\frac{G \nu_{e f f} K_{2}}{3 K_{3}^{2}} q z\left(z-\frac{d}{2}\right)(z-d), n_{y}=\frac{G \nu_{e f f}}{2 K_{3}} z(d-z)$.

This distortion of the director field can be detected optically by measuring the phase shift $\Phi_{\mathrm{H}}$ between the ordinary and the extraordinary components of a laser beam crossing the sample. In practice, the beam is never strictly perpendicular to the sample, which may be a source of error. For this reason, we calculated the phase shift at the compensation temperature by taking into account a small misalignment of the laser beam. Let $\theta$ be the angle (assumed to be small) between the beam and the normal to the sample and $\varphi$ the azimuthal angle of the beam with respect to the temperature gradient. A straightforward calculation yields

$$
\begin{aligned}
\Phi_{\mathrm{H}}= & -\left(\frac{G \nu_{e f f}}{K_{3}} d^{2}\right)^{2} \frac{n_{e}^{2}-n_{o}^{2}}{240 n_{e}^{2}} k n_{o} d \\
& +\frac{G \nu_{e f f}}{K_{3}} d^{2} \frac{n_{e}^{2}-n_{o}^{2}}{12 n_{e}^{2}} \theta(\sin \varphi) k d
\end{aligned}
$$

where $n_{o}$ and $n_{e}$ are the ordinary and extraordinary indices, respectively, $k=2 \pi / \lambda$ the angular wave number of the laser, while $\nu_{e f f}=\nu+K_{2} \frac{\mathrm{d} q}{\mathrm{~d} T}$ at $T_{c}$ as $q=0$. This equation shows that, at normal incidence $(\theta=0), \Phi_{H}$ is proportional to $G^{2}$ and $d^{5}$, a result already given by Éber and Jánossy in ref. [6]. On the other hand, an additional term linear in $G$ appears when the laser beam is slightly misaligned, but the term in $G^{2}$ remains unchanged. 
This experiment can be also performed in planar anchoring, provided that the molecular alignment direction be perpendicular to the temperature gradient. In this geometry, a nematic band forms which is centered on the inversion temperature. At equilibrium, this band is bordered by two $\chi$-disclination lines behind which regions twisted by $2 \pi$ form. As in the homeotropic case, the nematic is distorted by the temperature gradient. These distortions can be detected by measuring the phase shift $\Phi_{\mathrm{P}}$ between the ordinary and extraordinary components of a laser beam crossing the sample. A straightforward calculation gives in this case at the compensation temperature:

$$
\begin{aligned}
& \Phi_{\mathrm{P}}=-\psi+\left(\frac{G \nu_{e f f}}{K_{1}}\right)^{2} \frac{n_{e}^{2}-n_{o}^{2}}{240 n_{o}^{2}} k n_{e} d^{5}-\frac{G \nu_{e f f}}{K_{1}} \theta \\
& \times\left[\frac{n_{e}^{2}-n_{o}^{2}}{12 n_{o}^{2}} k d^{3} \sin \varphi+\left(\frac{d \cos ^{2} \frac{\psi}{2}}{k n_{o}^{2}}-\frac{\sin \psi}{k^{2} n_{o}^{2}\left(n_{e}-n_{o}\right)}\right) \cos \varphi\right]
\end{aligned}
$$

with $\psi=k d\left(n_{e}-n_{o}\right)$. As in the homeotropic case, the phase shift contains (in addition to the constant term $-\psi$ ) a linear term in $G$ which vanishes when $\theta=0$ and a quadratic term in $G$ proportional to $d^{5}$ and independent of angles $\theta$ and $\varphi$.

From these calculations, we see that measuring $\Phi_{\mathrm{H}}$ and $\Phi_{\mathrm{P}}$ on the one hand, and $n_{o}, n_{e}, K_{i}(i=1,2,3)$ and $\mathrm{d} q / \mathrm{d} T$ on the other hand, allow us to determine the Lehmann coefficient $\nu$. In the next section, we present our experiment and recall how to measure $\Phi_{\mathrm{H}}$ and $\Phi_{\mathrm{P}}$.

Experiment. - Our liquid crystal is a mixture of 4- $n$-octyloxy-4' cyanobiphenyl (8OCB from Synthon Chemicals $\mathrm{GmbH} \& \mathrm{Co}$ ) and of cholesteryl chloride (CC from Aldrich) in proportion 1:1 in weight with a compensation point at $59{ }^{\circ} \mathrm{C}$ and a clearing point at $67^{\circ} \mathrm{C}$. The $8 \mathrm{OCB}$ was purified by A. Zywocinsky and the CC was used without further purification. The glass plates were treated either for homeotropic anchoring with DMOAP (according to the Kahn procedure [12]) or for planar anchoring with a rubbed polyimide layer baked at $300{ }^{\circ} \mathrm{C}$ during 2 hours (ZLI 2650 from Merck). Nickel wires of calibrated diameters were used as a spacer to fix the sample thickness. The temperature gradient was imposed by placing the sample in a directional solidification cell (for its description, see ref. [13]), itself mounted on the stage of a polarizing microscope. A semi-reflecting plate was placed under the condensor of the microscope to illuminate the sample with a He-Ne laser $(\lambda=633 \mathrm{~nm})$. The laser beam was focused inside the sample with the condensor. Its diameter at the beam waist was of the order of $5 \mu \mathrm{m}$ and did not change significantly over the sample thickness (ranging between 50 and $110 \mu \mathrm{m}$ ). An $x$-y translation stage allowed manual positioning of he cell in order that the laser spot lies exactly in the middle of the nematic band, at the compensation temperature. The phase shift between

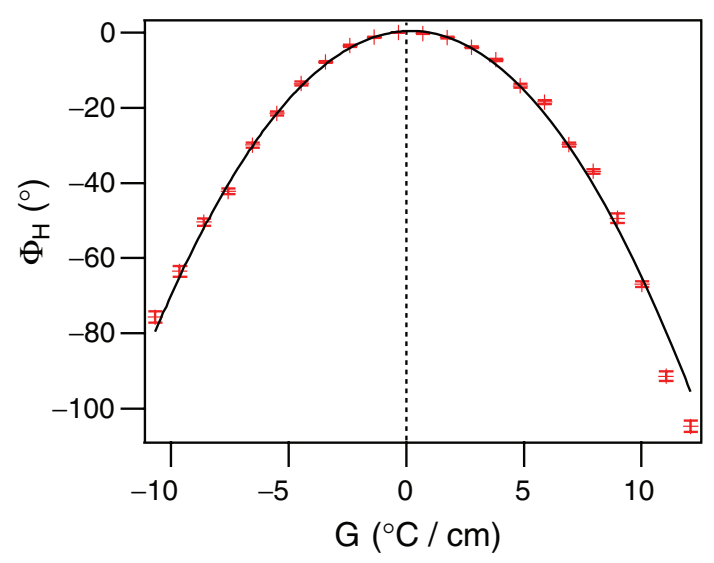

Fig. 1: Phase shift as a function of the temperature gradient ( $d=100 \mu \mathrm{m}$, homeotropic anchoring).

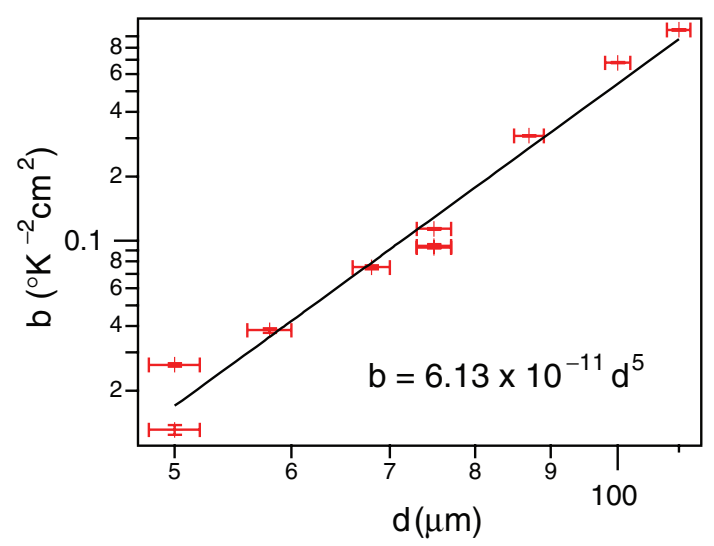

Fig. 2: Fit parameter $b$ as a function of the sample thickness $d$. The $d^{5}$-dependence is well satisfied.

the ordinary and extraordinary components of the laser beam crossing the sample was measured using a rotating analyzer, a quarter-wave plate, a photodiode and a lock-in amplifier following the method of Lim and Ho [14].

An example of curve obtained with a homeotropic $100 \mu \mathrm{m}$ thick sample is shown in fig. 1. The solid line represents the best fit of the data to a parabola $\Phi_{\mathrm{H}}=$ $a G-b G^{2}$. According to eq. (8), $a=\frac{\nu_{e f f}}{K_{3}} \frac{n_{e}^{2}-n_{o}^{2}}{12 n_{e}^{2}} \theta \sin \varphi k d^{3}$ and $b=\left(\frac{\nu_{e f f}}{K_{3}}\right)^{2} \frac{n_{e}^{2}-n_{o}^{2}}{240 n_{e}^{2}} k n_{o} d^{5}$. In fig. 2 , we plotted the fit parameter $b$ as a function of the sample thickness $d$. Within experimental errors, $b$ is proportional to $d^{5}$ as predicted by the theory. Fitting $b$ with a linear law in $d^{5}$ leads to $\left(\frac{\nu_{e f f}}{K_{3}}\right)^{2} \frac{n_{e}^{2}-n_{o}^{2}}{240 n_{e}^{2}} k n_{o}=$ $(9.3 \pm 0.8) \times 10^{13} \mathrm{rad} \mathrm{K}^{-2} \mathrm{~m}^{-3}$. As for values of $a$ obtained from the fits, they give expected typical angles $\theta \approx 1-3^{\circ}$.

The same procedure in planar geometry led to $\left(\frac{\nu_{e f f}}{K_{1}}\right)^{2} \frac{n_{e}^{2}-n_{o}^{2}}{240 n_{o}^{2}} k n_{e}=(25 \pm 6) \times 10^{13} \mathrm{rad} \mathrm{K}^{-2} \mathrm{~m}^{-3}$.

The next step was to measure the constant materials $n_{o}, n_{e}, K_{1}, K_{2}, K_{3}$ and $\mathrm{d} q / \mathrm{d} T$ at the compensation temperature. Our results are given in the next section. 


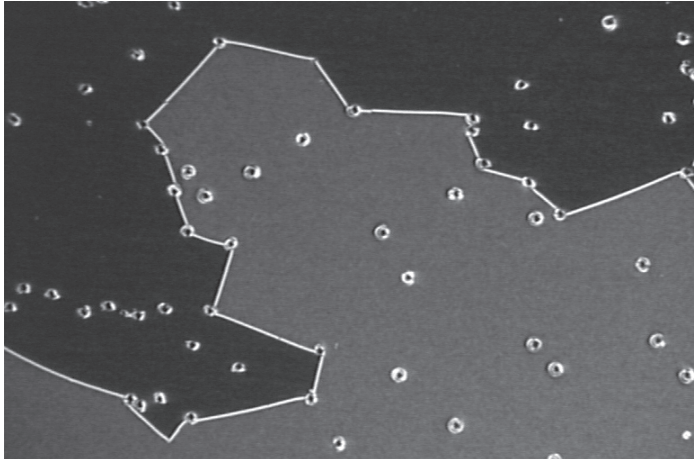

Fig. 3: Broken line separating an unwound zone (nematic phase, black) from a half-pitch twisted zone (grey). The line is pinned on the balls used to fix the sample thickness $(d=20 \mu \mathrm{m}$, $\left.T=59.6{ }^{\circ} \mathrm{C}\right)$.

Material constants. - Great care was taken to measure these coefficients (for the details, see the forthcoming longer article). The birefringence $\Delta n=n_{e}-n_{o}$ was obtained by measuring the transmitted intensity between crossed polarizers as a function of the local thickness in a wedge planar sample. The indices were then deduced separately by measuring in addition the phase shift between the ordinary and extraordinary components of a laser beam crossing homeotropic samples of different thicknesses at an incidence angle $\theta=15^{\circ}$. These two measurements gave $n_{o}=1.55 \pm 0.01$ and $n_{e}=1.64 \pm 0.01$.

The pitch was measured as a function of temperature by using commercial cells (from Instec, Inc) treated for planar anchoring. The cells contain silica balls which allow to fix their thickness accurately $(5,6.8,9$ or $20 \pm 0.2 \mu \mathrm{m})$. After filling, the cells were placed in a precision oven regulated within $0.01^{\circ} \mathrm{C}$. The absolute value of the pitch was measured by looking for the particular temperatures at which two zones with two different values of the twist coexist. Let $n$ and $n^{\prime}$ be the numbers of half pitches in two adjacent zones. It can be easily checked that the two zones have exactly the same elastic energy at temperature $T$ such that $q(T)=\frac{\left(n+n^{\prime}\right) \pi}{2 d}$. In general, $\left|n-n^{\prime}\right|=1$ (sometimes 2). Figure 3 shows an example of a $20 \mu \mathrm{m}$ thick sample in which an unwound zone (nematic phase, black between crossed polarizers) is in equilibrium with a half-pitch twisted zone. In this example, the temperature is adjusted in order that the $\chi$-line separating the two zones form a broken line joining the silica balls. By contrast, the line segments bend visibly in the direction of the zone of lower energy when the temperature is changed by typically $\pm 0.02{ }^{\circ} \mathrm{C}$. This method allowed us to measure $q(T)$ (fig. 4). The best fit of the experimental data to a polynomial of degree 2: $q=a\left(T-T_{c}\right)+b\left(T-T_{c}\right)^{2}$ led to $a=\frac{\mathrm{d} q}{\mathrm{~d} T}\left(T=T_{c}\right)=0.1365 \pm 0.001 \mu \mathrm{m}^{-1} \mathrm{~K}^{-1}$. The sign of $q$ was determined using a cell with a strong planar anchoring at the bottom plate and a gliding planar anchoring at the top plate.

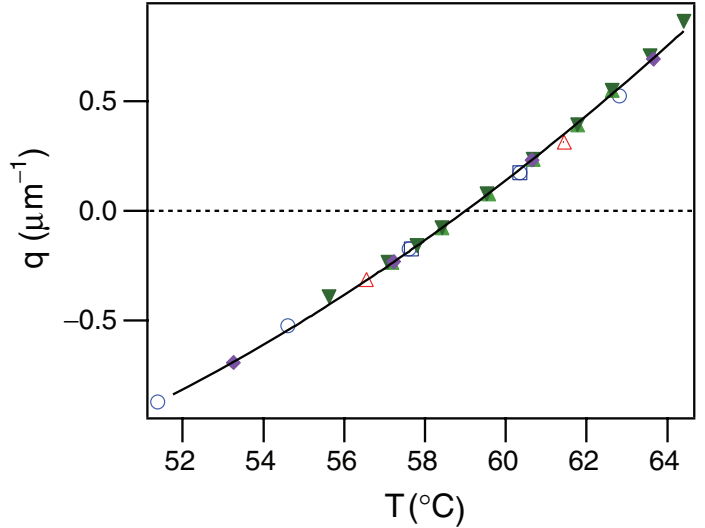

Fig. 4: Equilibrium twist as a function of temperature. The different symbols correspond to samples of different thicknesses $(5,6.8,9$ and $20 \mu \mathrm{m})$.

In order to measure the elastic constants, we used three different methods. First, we measured the Fréedériks transition in planar samples using a capacitive method. From this experiment (and capacity measurements of homeotropic samples) we deduced the dielectric constants $\left(\varepsilon_{\|}=9.4 \pm 0.5\right.$ and $\left.\varepsilon_{\perp}=4.5 \pm 0.2\right)$ and the ratios $\frac{K_{1}}{\varepsilon_{0} \varepsilon_{a}}=$ $0.076 \pm 0.006 \mathrm{~V}^{2}$ (with $\varepsilon_{a}=\varepsilon_{\|}-\varepsilon_{\perp}$ ) and $\frac{K_{3}}{K_{1}}=1.7 \pm 0.1$. The latter was deduced from the fit of the whole capacity vs. voltage curve according to the procedure given in [15] by neglecting flexoelectric effects [16] (this point will be justified in a forthcoming article). Second, we measured the Fréedériks transition in a $\pi / 2$-twisted planar cell (thickness $6.8 \mu \mathrm{m}$, from Instec, Inc) close to the compensation point. Measuring the critical voltage as a function of the temperature (or, equivalently, as a function of $q$ ) gave us $\frac{K_{2}}{\varepsilon_{0} \varepsilon_{a}}=0.068 \pm 0.002 \mathrm{~V}^{2}$ [17]. Finally, we measured the spinodal voltage of the nematic phase in homeotropic samples of various thicknesses as a function of the temperature (or $q$ ). These measurements led to $\frac{K_{3}}{\varepsilon_{0} \varepsilon_{a}}=0.15 \pm 0.02 \mathrm{~V}^{2}$ and $\frac{K_{2}^{2}}{K_{3} \varepsilon_{0} \varepsilon_{a}}=0.0312 \pm 0.0006 \mathrm{~V}^{2}$.

Lehmann coefficient. - From the measurements described in the two previous sections, we can calculate all the materials constants, including the Lehmann coefficient $\nu$. All the measurements being coupled, the error estimation is not simple and was determined by using the maximum-likelihood method described in ref. [18]. Finally, we found for the elastic constants: $K_{1}=(3.4 \pm 0.4) \times 10^{-12} \mathrm{~N}, \quad K_{2}=(2.8 \pm 0.2) \times 10^{-12} \mathrm{~N}$ and $K_{3}=(5.9 \pm 0.6) \times 10^{-12} \mathrm{~N}$, and for the Lehmann coefficient:

$$
\nu=(2.8 \pm 0.6) \times 10^{-7} \mathrm{~kg} \mathrm{~K}^{-1} \mathrm{~s}^{-2} .
$$

In conclusion, we have found, in agreement with the pioneer work of Éber and Jánossy, that the Lehmann coefficient does not vanish at the compensation temperature in a cholesteric liquid crystal. This result is in favor of 


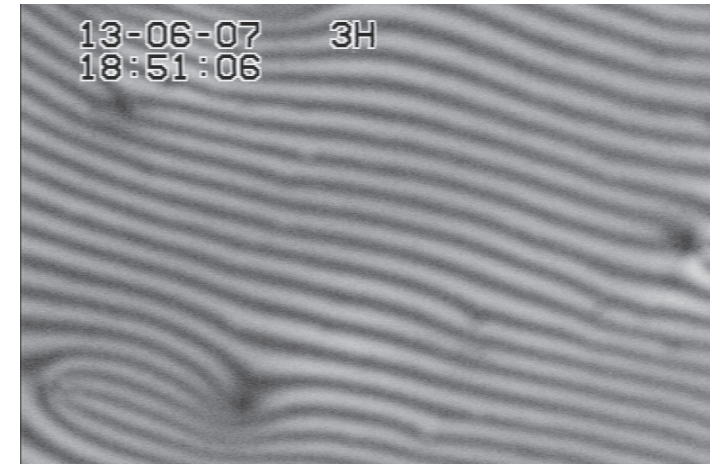

Fig. 5: Banded texture formed after a sample treated for gliding planar anchoring on both plates was submitted during a few hours to a vertical temperature gradient $(d=10 \mu \mathrm{m})$.

a microscopic origin of the Lehmann effect, instead of a structural one, as proposed by Pleiner and Brand. This result is also coherent with a recent experiment on the chemical Lehmann effect in a Langmuir monolayer [19]. Indeed, the rotation of the director is here due to a flux of water molecules across a single layer of chiral molecules and does not involve a macroscopic helix.

We emphasize that we determined this coefficient in two different geometries (homeotropic, but also planar, which is new) and that our measurements of the material constants were often redundant, but always compatible with one another. In addition, the two experiments yielded similar results as the one in homeotropic anchoring gives alone $\nu / \varepsilon_{0} \varepsilon_{a}=(7 \pm 1.3) \times 10^{3} \mathrm{~V}^{2} \mathrm{~K}^{-1} \mathrm{~m}^{-1}$, while the other one in planar anchoring (less accurate) gives $\nu / \varepsilon_{0} \varepsilon_{a}=$ $(4.9 \pm 2.6) \times 10^{3} \mathrm{~V}^{2} \mathrm{~K}^{-1} \mathrm{~m}^{-1}$.

A well-informed reader could also object that our results contradict those of Madhusudana et al. [20,21] about the electric Lehmann effect at the compensation point. For this reason, we redid their experiment with our mixture. Although our observations were essentially the same as theirs, we showed that they could not result from an electric Lehmann effect, but more simply from flexoelectricity. These results will be published in a future article [22].

Finally, we would like to mention that we recently observed a continuous Lehmann rotation of the director at the compensation temperature in a sample treated on both sides for planar gliding anchoring. In this experiment, the sample was placed in a temperature gradient perpendicular to the glass plates. After a few hours in the temperature gradient, the sample, initially homogeneous, developed a banded texture between crossed polarizers (fig. 5) analogous to that calculated numerically by theorists $[23,24]$, revealing a rotation of several turns of the director. This preliminary experiment thus confirms the results given in the article.

\section{$* * *$}

We thank A. ZYwOCinsKY for the purification of the liquid crystal $8 \mathrm{OCB}$ and Sriram Ramaswamy for helpful discussion.

\section{REFERENCES}

[1] Lehmann O., Ann. Phys. (Leipzig), 2 (1900) 649.

[2] Lehmann O., Flüssige Kristalle und ihr Scheinbares Leben (Verlag von Leopold Voss, Leipzig) 1921.

[3] Leslie F. M., Proc. R. Soc. London, Ser. A, 307 (1968) 359.

[4] De Gennes P.-G. and Prost J., The Physics of Liquid Crystals (Oxford University Press, Oxford) 1995.

[5] Oswald P. and Pieranski P., Nematic and Cholesteric Liquid Crystals: Concepts and Physical Properties Illustrated by Experiments (Taylor \& Francis, CRC press, Boca Raton) 2005.

[6] Éber N. and Jánossy I., Mol. Cryst. Liq. Cryst. Lett., 72 (1982) 233.

[7] Éber N. and Jánossy I., Mol. Cryst. Liq. Cryst. Lett., 102 (1984) 311.

[8] Pleiner H. and Brand H. R., Mol. Cryst. Liq. Cryst. Lett., 5 (1987) 61.

[9] Pleiner H. and Brand H. R., Mol. Cryst. Liq. Cryst. Lett., 5 (1988) 183.

[10] Éber N. and Jánossy I., Mol. Cryst. Liq. Cryst. Lett., 5 (1988) 81.

[11] Oswald P., Baudry J. and Pirkl S., Phys. Rep., 337 (2000) 67.

[12] Kahn F. J., Appl. Phys. Lett., 22 (1973) 386.

[13] Oswald P., Moulin M., Metz P., Géminard J.-C. and Sotta P., J. Phys. III, 3 (1993) 1891.

[14] Lim K. C. and Ho J. T., Mol. Cryst. Liq. Cryst., 47 (1978) 173.

[15] Morris S. W., Palffy-Muhoray P. and Balzarini D., Mol. Cryst. Liq. Cryst., 139 (1986) 263.

[16] Brown C. V. and Mottram N. J., Phys. Rev. E, 68 (2003) 031702.

[17] Raynes E. P., Mol. Cryst. Liq. Cryst. Lett., 4 (1986) 1.

[18] Taupin D., Probabilities Data Reduction and Error Analysis in the Physical Sciences (Les Editions de Physique, Les Ulis) 1988.

[19] Tabe Y. and Yokoyama H., Nat. Mater., 2 (2003) 806.

[20] Madhusudana N. V., Pratibha R. and Padmini H. P., Mol. Cryst. Liq. Cryst., 202 (1991) 35.

[21] Padmini H. P. and Madhusudana N. V., Liq. Cryst., 14 (1993) 497.

[22] Dequidt A. and Oswald P., Does electric Lehmann effect exist in cholesteric liquid crystals?, to be published in Eur. Phys. J. E.

[23] Tsori Y. and De Gennes P.-G., Eur. Phys. J. E, 14 (2004) 91.

[24] Svenšek D., Pleiner H. and Brand H. R., Phys. Rev. Lett., 96 (2006) 140601. 